\title{
Breast Cancer Surveillance Guidelines
}

\author{
By Thomas J. Smith, MD, FACP, FASCO
}

Johns Hopkins Medical Institutions, Baltimore, MD

The American Society of Clinical Oncology (ASCO) has updated the 2006 version of its breast cancer surveillance guidelines. In short, there are no changes to the guidelines. It is worth reviewing the gist of the guidelines, as summarized in Table 1, compared with what oncologists reported they do in a survey. The low averages do not adequately represent the true variation-we all know oncologists who routinely perform these tests. Unpublished reports show some $30 \%$ to $40 \%$ of practices routinely measure serum tumor markers and perform computed tomography or positron emission tomography scans. The data are hard to find, given that practitioners have not been supposed to do these tests for a decade.

\section{What Is Behind the Practice of Too-Active Surveillance?}

This practice is not driven by profit motives. Grunfeld et $\mathrm{al}^{2}$ studied practice patterns in Ontario and found that one quarter of asymptomatic women were undergoing imaging such as computed tomography or bone scans, and there was no way for the physicians involved to own their facilities or profit from the tests.

The practice is not driven by lack of time to discuss the guidelines and give the evidence against tests. I used a stopwatch with consecutive patients and found that the average discussion of the guidelines was approximately 6 minutes, if I had a printed copy on which to write. ${ }^{3}$

My understanding is that aggressive surveillance testing results from several things. First, it is hard to tell a patient that even if we find his or her cancer sooner, it will not make a difference in curability or even survival time-particularly if the oncology group competition does perform the tests.

Second, some physicians believe that the newer drugs for breast cancer are so much better that we can give people longer and better survival if we find their disease sooner. There is to date absolutely no evidence that any of the newer drugs or radiation therapies lead to more cures than doxorubicin or the taxanes available when the randomized surveillance trials were conducted. There is no evidence that people live longer when they have a single asymptomatic liver breast cancer metastasis ablated by whatever means. We all know that human epidermal growth factor receptor 2-directed therapy leads to longer survival in breast cancer, and we hope for better therapy for the rest. Those of us who support the evidence-based guidelines could just as effectively argue that better therapies will allow us to salvage a patient found with a $1.6-\mathrm{cm}$ symptomatic bone metastasis instead of a $1-\mathrm{cm}$ asymptomatic metastasis. We have always demanded evidence to change our practice, and surveillance should be no exception.

These are not trivial issues. If there are 1,000,000 female breast cancer survivors, requiring each to undergo a positron emission tomography scan every other year and a cancer antigen 27.29 test once a year will be an absolute waste of a lot of money, not to mention the additional radiation exposure and the opportunity cost of using those billions of dollars for unhelpful tests when newer drugs are available. With current projections that half of all household income will be dedicated to health care by $2017,{ }^{4}$ we clearly must find ways to reduce costs without compromising quality too much.

\section{Getting to "No"}

ASCO has recognized the wastefulness of these practices and placed the reduction of unhelpful surveillance on its top-five list of oncology goals. ${ }^{5}$ This will give insurance companies the scientific background and administrative gumption they need to decline payment for these tests. Already, Medicare does not pay for the tumor marker tests. Practice-by-practice and practitioner comparisons will allow us to monitor our practices with the Quality Oncology Practice Initiative 6 and identify the practices in which $40 \%$ of women undergo serum tumor

Table 1. Guideline Dos and Don'ts and Actual Practice

\begin{tabular}{lll}
\hline & Recommendation & Actual Practice $^{1}$ \\
\hline Do & History and physical examination every 3 to 6 months for 3 years, then every 6 to 12 & 3.4 in year 1, falling to two in year 5 \\
& months for the next 2 years, then annually & \\
& Yearly mammogram & 1.6 in year 1, falling to 1.3 in year 2 \\
Don't do & Routine blood tests such as CBC, LFT & Two \\
& Serum tumor markers & 0.4, CEA; 0.7, CA 15.3 \\
& Chest x-rays, CTs, bone scans, PET scans in patients without symptoms & 0.1 for all with SD of 0.4 to 0.6 \\
\hline
\end{tabular}

Abbreviations: CA, cancer antigen; CBC, complete blood count; CEA, carcinoembryonic antigen; CT, computed tomography; LFT, liver function test; PET, positron emission tomography; SD, standard deviation. 
Table 2. ASCO Recommendations for Breast Surveillance, and Verbal and Written Comments*

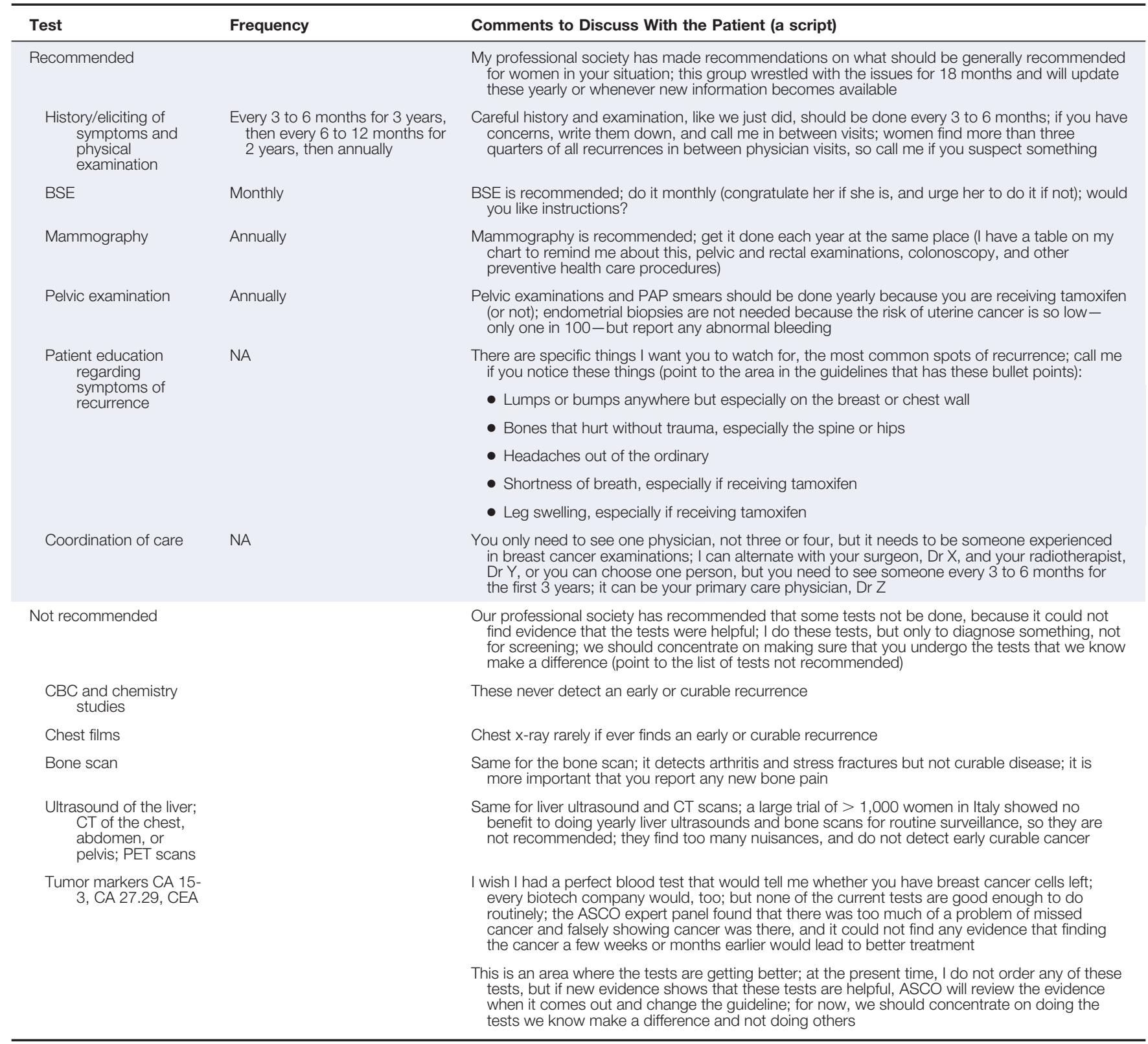

Abbreviations: ASCO, American Society of Clinical Oncology; BSE, breast self-examination; CA, cancer antigen; CBC, complete blood count; CEA, carcinoembryonic antigen; CT, computed tomography; NA, not applicable; PAP, Papanicolaou; PET, positron emission tomography.

* I write directly on a printed copy of the surveillance guidelines and fax a copy to the primary care physician.

marker tests. The Quality Oncology Practice Initiative must become a "diminish overuse" program as much as an "ensure appropriate use" program to stay relevant, and these changes are under way.

Ultimately, however, we need a new script for explaining this to people in a way that makes sense to them. ${ }^{7}$ I use the script in Table 2, writing on the printed guidelines, ${ }^{8}$ and it is very doable in a single visit. Once I tell people about what we should not do, I can write a list of what we should do- exercise, maintaining baseline weight, health maintenance for other problems, a good vegetable-based diet, and consideration of an aspirin a day. ${ }^{9}$

\section{Author's Disclosures of Potential Conflicts of Interest}

The author(s) indicated no potential conflicts of interest.

Corresponding author: Thomas J. Smith, MD, FACP, Harry J. Duffey Family Professor of Palliative Medicine, Johns Hopkins Medical Institutions, Professor of Oncology, Sidney Kimmel Comprehensive Cancer Center, 600 N. Wolfe St, Blalock 369, Baltimore, MD 21287-0005; e-mail: tsmit136@jhmi.edu. 


\section{References}

1. Margenthaler JA, Allam E, Chen L, et al: Surveillance of patients with breast cancer after curative-intent primary treatment: Current practice patterns. J Oncol Pract 8:79-83, 2012

2. Grunfeld E, Hodgson DC, Del Giudice ME, et al: Population-based longitudinal study of follow-up care for breast cancer survivors. J Oncol Pract 6:174-181, 2010

3. Smith TJ: American Society of Clinical Oncology recommended breast cancer surveillance guidelines can be done in a routine office visit. J Clin Oncol 23:6807, 2005

4. Young RA, DeVoe JE: Who will have health insurance in the future? An updated projection. Ann Fam Med 10:156-162, 2012

5. Schnipper LE, Smith TJ, Raghavan D, et al: American Society of Clinical
Oncology identifies five key opportunities to improve care and reduce costs: The top five list for oncology. J Clin Oncol 30:1715-1724, 2012

6. Jacobson JO, Neuss MN, McNiff KK, et al: Improvement in oncology practice performance through voluntary participation in the Quality Oncology Practice Initiative. J Clin Oncol 26:1893-1898, 2008

7. Loprinzi CL, Hayes D, Smith T: Doc, shouldn't we be getting some tests? $\mathrm{J}$ Clin Oncol 18:2345-2348, 2000

8. Cancer.net: What to know: ASCO's guideline on follow-up care for breast cancer. http://www.cancer.net/publications-and-resources/what-know-ascosguidelines/what-know-ascos-guideline-follow-care-breast-cancer

9. Smith TJ, Snyder C: Is it time for (survivorship care) plan B? J Clin Onco 29:4740-4742, 2011

\section{JOP Web Site Optimized for Mobile Platforms}

Connect on the go with your iPhone, iPod touch, and Android devices. The interface is designed for quick navigation and enabled to automatically appear on your mobile device, so you can conveniently access JOP's cutting-edge research from any location. Log on now at 